\title{
Design of Optical Logic Gates Using Self-collimated Beams in 2D Photonic Crystal
}

\author{
X. Susan CHRISTINA ${ }^{1}$ and A. P. KABILAN ${ }^{2}$ \\ ${ }^{1}$ Department of ECE, Mookambigai College of Engineering, Trichy, Tamilnadu, India \\ ${ }^{2}$ Chettinad College of Engineering \& Technology, Puliyur, Tamiladu, India \\ *Corresponding author: X. Susan CHRISTINA E-mail: fab_jesu@yahoo.co.in
}

\begin{abstract}
Optical logic gates are elementary components for optical network and optical computing. In this paper, we propose a structure for AND, NAND, XNOR and NOR logic gates in the two dimensional photonic crystal which utilizes the dispersion based self-collimation effect. The self-collimated beam is splitted by the line defect and interfered with other self-collimated beam. This interference may be constructive or destructive based on their phase difference. This phenomenon is employed to realize all-optical logic gates. The gates are demonstrated numerically by computing electromagnetic field distribution using the finite difference time domain (FDTD) method. The results ensure that this design can function as AND, NAND, XNOR and NOR logic gates. The size of the structure is about $10 \mu \mathrm{m} \times 10 \mu \mathrm{m}$ which in turn results in an increase in the speed and all the gates are realized in the same configuration. The ON-OFF contrast ratio is about $6 \mathrm{~dB}$.
\end{abstract}

Keywords: Optical computing, photonic crystal, band diagram, equifrequency contour, all-optical logic gates

\section{Introduction}

The development of the all-optical technology is vital to realize the future telecommunication network and optical computer, where all the functions could be carried out in the optical domain. All-optical logic gates are the key elements in optical digital information processing. Ultrafast optical AND gate devices have been proposed to increase the bit rate of an optical transmission system and to realize an optical time division demultiplexer [1]. In an optical packet switching network, the photonic routers are expected to handle XOR operations for optical label swapping and header reconfiguration $[2,3]$. So the realization of various logic operations for ultrafast signals is essential for future applications. Recently, some works were reported on all-optical logic circuits. But most of the reported works suffered from some certain fundamental limitations including big size, low speed and the difficulty in performing chip-scale integration. In the literature, some schemes of all-optical logic gates were reported by use of nonlinear effects in optical fibers [4-6], in semiconductor devices [7-11], and in waveguides [12-14].

Photonic crystal $(\mathrm{PhC})$ structures have been extensively studied recently because of their unique properties such as compactness, high speed, low power consumption, better confinement and the promise in photonic integrated circuits $[15,16]$. $\mathrm{PhC}$ is a kind of nanostructures with a periodic modulation of the permittivity. Logic functions based on the photonic crystal can be realized by nonlinear effects [17], ring resonator [18], and multimode interference [19]. These require 
significant amount of the power for the nonlinear material, long interaction length and two different wavelengths for probing and input signals. The complex spatial dispersion property of the self-collimation effect in the $\mathrm{PhC}$ provides a mechanism to employ logic functions. In our previous work [20], OR and XOR logic functions were realized by applying only two input signals. In this paper, we propose other logic gates AND, NAND, XNOR, and NOR by applying the reference signal along with input signals with the same wavelength. Its configuration is simple and compact.

The paper is organized as follows: in Section 2, the self-collimation effect and band diagram of the proposed structure is explained; the structure of logic gates and optimum values are described in Section 3; in Section 4, the operation of logic gates on the platform of two dimensional (2D) $\mathrm{PhC}$ is investigated; the conclusions are drawn in Section 5.

\section{Self-collimation effect and band diagram}

One of the interesting confinement mechanisms of light guiding in the photonic crystal is selfcollimation effect. It exploits the spatial dispersion properties of Bloch waves to achieve the electromagnetic wave without diffraction. It retains spatial width confinement without the line defect waveguide or nonlinearities [21]. The shape of the $\mathrm{PhC}$ dispersion surface is an essential feature for determining the self-collimation. This can be optimized and manipulated by lattice symmetry, material parameters and the geometry shape of the constituents.

A square lattice of the dielectric rod based 2D silicon $\mathrm{PhC}$ structure is considered here. The permittivity $\left(\varepsilon_{r}\right)$ and the refractive index of the silicon rods are 11.97 and 3.46, respectively. The radius of the host rod $(r)$ is taken as $105 \mathrm{~nm}$. The most notable specification of the $\mathrm{PhC}$ is its photonic band gap. In the photonic band gap, the light with certain range of frequencies is not permitted to propagate inside the crystal. Using the plane wave expansion (PWE) method, the energy band diagram and equifrequency contour (EFC) for E-polarized mode-electric-field is calculated and demonstrated in Figs. 1(a) and 1(b). This field is parallel to the axes of the rod.

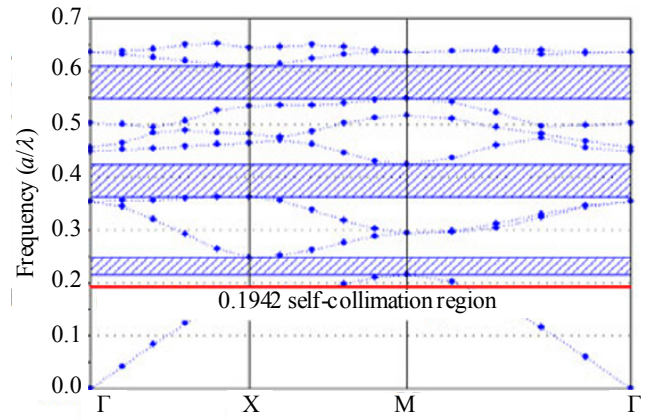

(a)

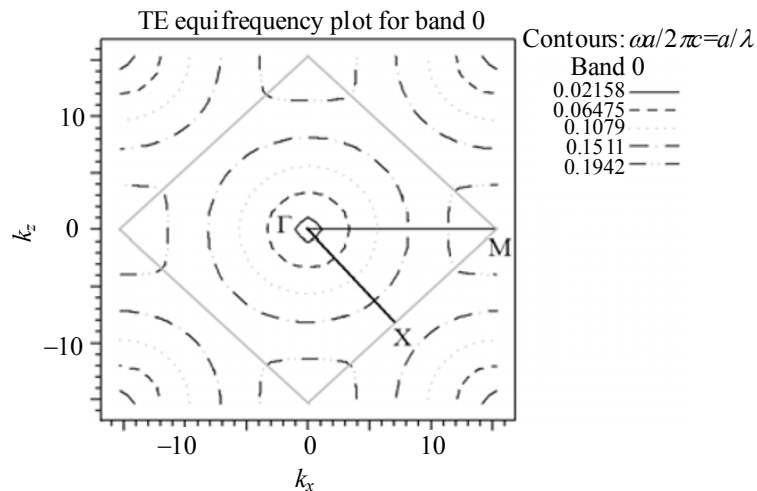

(b)

Fig. 1 Band structure and contour map: (a) band diagram of the square lattice PC structure for E-polarized mode and (b) equifrequecy contours of the $\mathrm{PhC}$ dispersion surface in the first Brillouin zone.

In the band diagram, $x$ axis is divided into regions representing the line segments connecting the $\Gamma-\mathrm{X}-\mathrm{M}-\Gamma$ points in the wave vector space corresponding to $(0,0),(\sqrt{ } 2 \pi / a, 0)$ and $(\sqrt{ } 2 \pi / a$, $\sqrt{2} \pi / a$ ), respectively. In the EFCs, the curves of the frequencies around $0.194(a / \lambda)$ can be identified as squares with round corners centered at $\mathrm{M}$ point, where $\lambda$ is the wavelength of the incident radiation whose value is $1555.1 \mathrm{~nm}$. In the flat square, the direction of light propagation in the $\mathrm{PhC}$ is identical to the direction of the group velocity given by $v_{\mathrm{g}}=\nabla_{\mathrm{k}} \omega(k)$, where $\omega(k)$ is the optical frequency at the wave vector $k$. It means that the group velocity is perpendicular to the EFCs, and the waves are 
collimated. Hence, the self-collimation phenomenon occurs, when the E-polarized light of the frequencies around $0.194(a / \lambda)$ propagates along the $\Gamma-\mathrm{M}$ direction.

\section{Structure of logic gates and its optimum value}

Figure 2(a) exemplifies the 2D PhC lattice used for designing logic gates. The circles represent the silicon rods whose radii are $105 \mathrm{~nm}$. The distance between two rods which is known as the lattice constant " $a$ " is $302 \mathrm{~nm}$. In this structure two line defects are created by reducing the radius of 15 rods in the $\Gamma-\mathrm{X}$ direction, and the distance between them is " $10 a$ ". When the self-collimated beam is incident on the line defect, it is partially reflected and partially transmitted. The power splitting ratio at the line defect and phase difference between the transmitting and reflecting signals are depending on the radius of the defect rod.

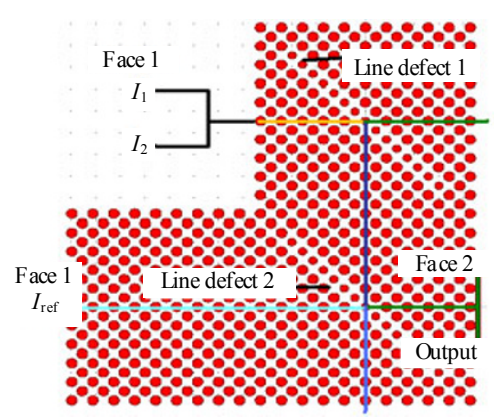

(a)

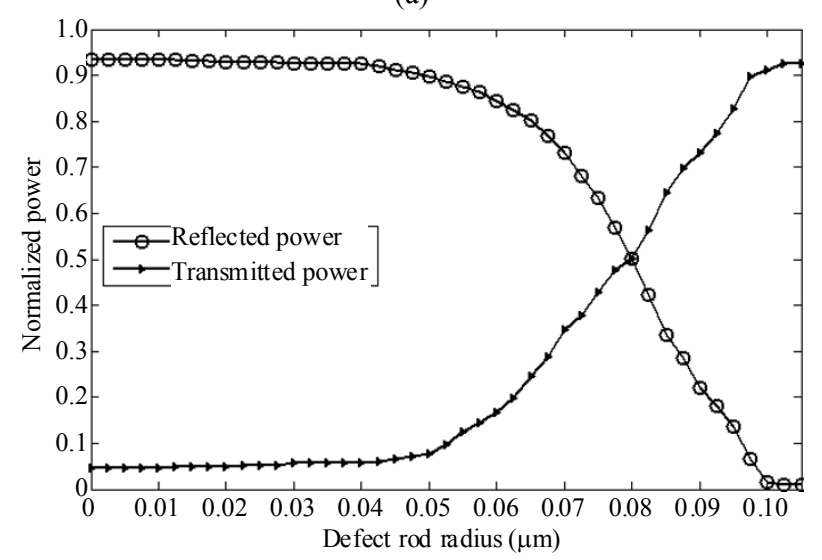

(b)

Fig. 2 Logic gate structure and power ratio: (a) the proposed structure of logic gates and (b) the normalized transmitted power and reflected power with respect to the defect rod radius.
From Fig. 2(b), it is evident that when the radius $\left(r_{d}\right)$ of the defect rod radius is $83 \mathrm{~nm}$, the transmitting power and the reflecting power are divided equally. If the radii of the defect rods are greater than those of host rods, the phase difference between the transmitting signal and the reflecting signal is " $-\pi / 2$ ", whereas if it is less than those of host rods, the phase difference is " $\pi / 2$ " [22].

The amplitudes of the transmission signal and

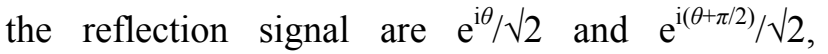
respectively. When two self-collimated signals with appropriate phase difference are introduced at the line defect, these signals are interfered either constructively or destructively. The intensities of constructive and destructive signals are $2|u E|^{2}$ and 0 , respectively, where $E$ represents plane wave and $u$ is a periodicity function of the $\mathrm{PhC}$.

The transmitted signal and reflected signal are expressed as

$$
\begin{gathered}
R_{12}=\frac{u E}{\sqrt{2}} \mathrm{e}^{\mathrm{i}(\theta+\pi)} \\
T_{22}=\frac{u E}{\sqrt{2}} \mathrm{e}^{\mathrm{i} \theta}
\end{gathered}
$$

where $R_{12}$ is the reflected input signal 1 at line defect 2 , and $T_{22}$ is the transmitted input signal 2. The resultant signal at the output port is a linear combination of the reflected beam and transmitted beam, expressed as

$$
O=R_{12}+T_{22}=\frac{u E}{\sqrt{2}}\left(\mathrm{e}^{\mathrm{i}(\theta+\pi)}+\mathrm{e}^{\mathrm{i} \theta}\right),
$$

and its corresponding intensity is

$$
I=|O|^{2}=\left|\frac{u E}{\sqrt{2}}\left(\mathrm{e}^{\mathrm{i}(\theta+\pi)}+\mathrm{e}^{\mathrm{i} \theta}\right)\right|^{2} .
$$

\section{Operation of logic gates on the platform of the 2D PhC}

To demonstrate the functions of logic gates, the numerical experiment is performed. Two Gaussian TM polarized input optical signals $\left(I_{1}\right.$ and $\left.I_{2}\right)$ are launched at the line defect 1 , and the reference optical signal $\left(I_{\text {ref }}\right)$ is launched at the line defect 2. The wavelength of the input and the reference signals is set to be $1555.1 \mathrm{~nm}$. The path lengths of 
the input signal and reference signal are equal in length. The reflected input beams $I_{1} \& I_{2}$ and transmitted reference beam $I_{\text {ref }}$ are interfered at the line defect 2 . This interference is either constructive or destructive according to their phase difference. The destructive output signal is taken out from the face 2 .

For AND logic simulation, the input signals $I_{1} \&$ $I_{2}$ and reference intensities $I_{\text {ref }}$ are taken as $2 I_{0}$ and $0.5 I_{0}$, respectively. Input and the reference signals are interfered constructively or destructively at the line defect 2. The destructive interfered signal is consider as an output at the output port A. When none of the input signal is given, only $I_{\text {ref }}\left(0.5 I_{0}\right)$ is incident on the line defect 2 , and it is divided equally.
So the output intensity is " $0.25 I_{0}$ ". If any one of the inputs is high, the input signal $\left(2 I_{0}\right)$ is divided equally as the transmitted and reflected beams by the line defect 1 . The reflected input beam $\left(I_{0}\right)$ will be incident on the line defect 2 . In this line defect 2 , the reflected input is again divided and interfered with the transmitted reference beam. It is found that the output intensity is " $0.25 I_{0}$ ". When both the input signals are applied, the reflected beam from the line defect 1 is $2 I_{0}$. At the second line defect, the reflected input beam $\left(I_{0}\right)$ destructively is interfered with the transmitted reference beam $\left(0.5 I_{0}\right)$, and the output is " $0.75 I_{0}$ ". The field distribution at the steady state operation of the AND logic gate is illustrated in Fig. 3.

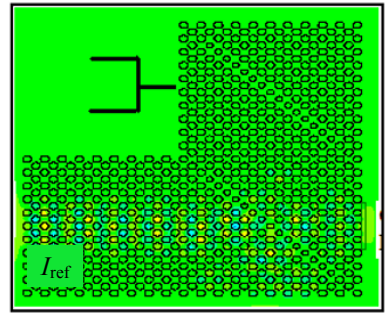

(a)

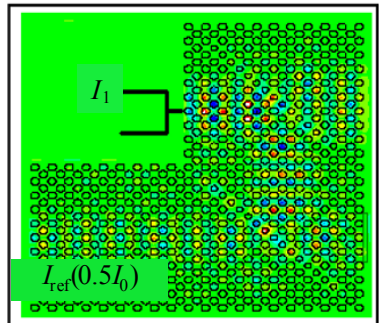

(b)

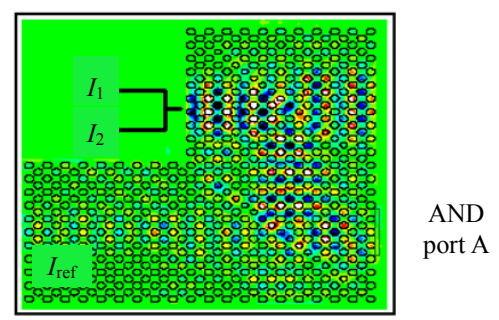

(c)

Fig. 3 Electromagnetic field distribution for the AND logic: (a) both inputs are low, (b) any one of the inputs is high, and (c) both inputs are high.

In the NAND gate analysis, input and reference signals are set as $I_{1}=I_{2}=I_{\text {ref }}=2 I_{0}$, and the behavior of the NAND logic gate is shown in Fig. 4 for various input combinations. When none of the input signals is given, $I_{\text {ref }}\left(2 I_{0}\right)$ is incident on the line defect 2 . The transmitted signal of the reference signal " $I_{0}$ " is considered as the output. If any one of the signals is high either 01 or 10 , the partially reflected input beam $\left(0.5 I_{0}\right)$ is destructively interfered with the transmitted reference signal $\left(I_{0}\right)$ at the line defect 2 , and the resultant output is " $0.5 I_{0}$ ". If both the inputs are high, the output is zero since reflected input signals $\left(I_{0}\right)$ are destructively interfered with the transmitted reference signal $\left(I_{0}\right)$.

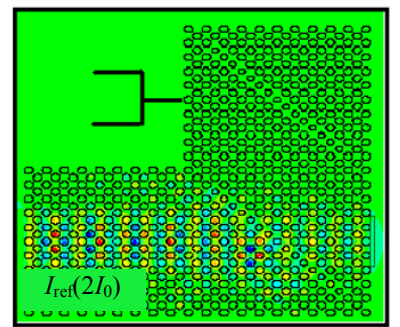

(a)

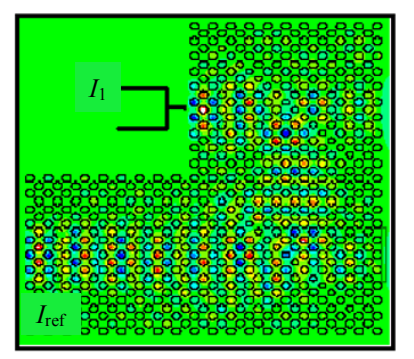

(b)

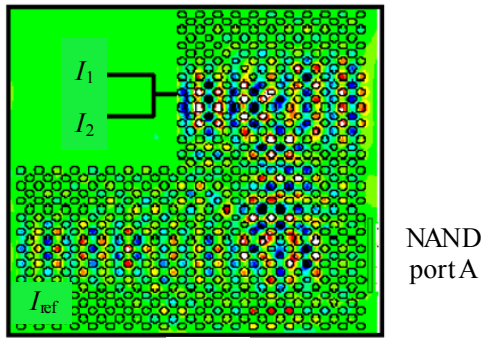

(c)

Fig. 4 Electromagnetic field distribution for NAND logic: (a) both inputs are low, (b) any one of the inputs is high, and (c) both inputs are high.

In the case of the NOR logic operation, the excited simultaneously, the partially reflected input intensities of input and the reference signals are $I_{1}=$ $I_{2}=I_{\text {ref }}=I_{0}$. When both the input signals $I_{1}$ and $I_{2}$ are beams $\left(0.5 I_{0}\right)$ are destructively interfered with the transmitted reference signal $\left(0.5 I_{0}\right)$ at the line defect 
2 , and the output is " 0 ". If none of the inputs is applied, the transmittance reference signal is taken as the output, and its value is " $0.5 I_{0}$ ". If any one of the input signals is incident, the reflected input beam $\left(0.25 I_{0}\right)$ is interfered with the transmitted reference signal $\left(0.5 I_{0}\right)$, and the output is " $0.25 I_{0}$ ". Figure 5 shows the electromagnetic field distribution of various input combinations of the NOR gate.

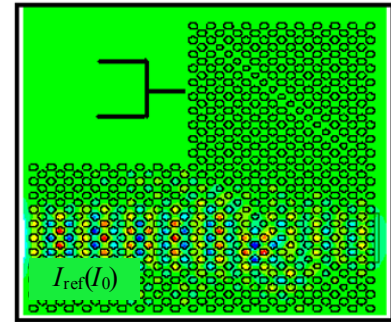

(a)

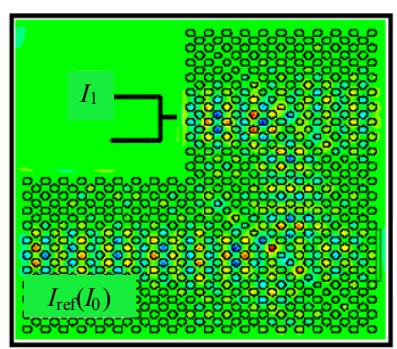

(b)

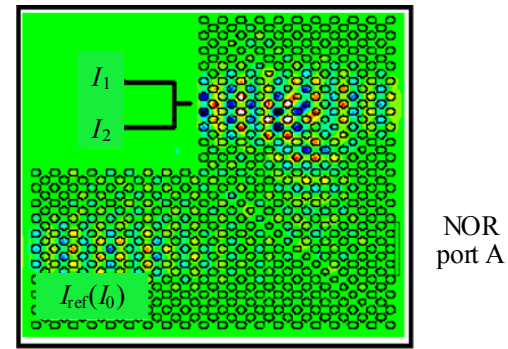

(c)

Fig. 5 Field distribution for NOR logic: (a) both inputs are low, (b) any one of the inputs is high, and (c) both inputs are high.

The operation of the XNOR logic gate is explained as follows: input signals and the reference signal are taken as $2 I_{0}$ and $I_{0}$, respectively. When both the input signals $I_{1}$ and $I_{2}$ are low, only the reference signal is divided at the line defect 2 , and the transmittance reference signal " $0.5 I_{0}$ " is considered as the output. If both the signals are high,

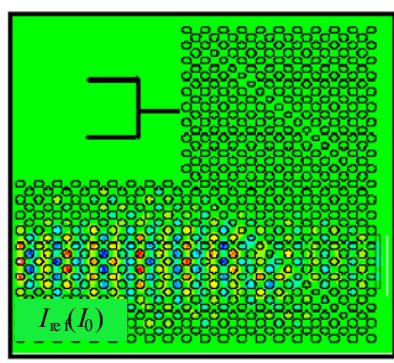

(a)

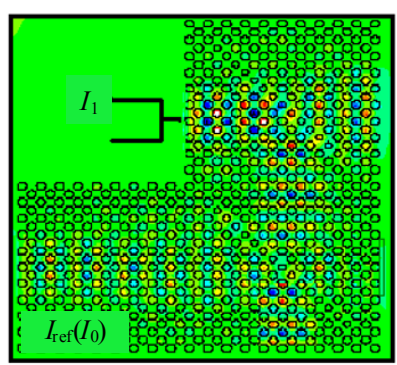

(b)

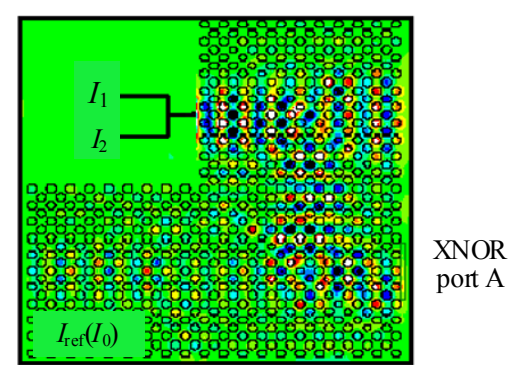

(c)

Fig. 6 Electromagnetic field distribution for XNOR logic: (a) both inputs are low, (b) any one of the inputs is high, and (c) both inputs are high.

Numerically simulated output signal levels for all the proposed logic gates are shown in Table 1. The output values of logic gates can be standardized using the threshold limiter which suppresses " 0 " level signals and stabilizes fluctuations of "1"

Table 1 Output signal level for proposed gates.

\begin{tabular}{ccccc}
\hline Input & AND & NAND & XNOR & NOR \\
\hline 00 & $0.25 I_{0}(0)$ & $I_{0}(1)$ & $0.5 I_{0}(1)$ & $0.51 I_{0}(1)$ \\
01 & $0.24 I_{0}(0)$ & $0.5 I_{0}(1)$ & $0.095(0)$ & $0.25 I_{0}(0)$ \\
10 & $0.24 I_{0}(0)$ & $0.5 I_{0}(1)$ & $0.01(0)$ & $0.25 I_{0}(0)$ \\
11 & $0.75 I_{0}(1)$ & $0.09 I_{0}(0)$ & $0.5 I_{0}(1)$ & $0(0)$ \\
\hline
\end{tabular}

level signals.

The ON-OFF contrast ratio of the gates is defined as the logarithmic ratio of the $\mathrm{ON}$ and $\mathrm{OFF}$ power level. The contrast ratios for the exact simulated value are tabulated in Table 2 .

Table 2 ON-OFF contrast ratio for various logic gates.

\begin{tabular}{cc}
\hline Gate & ON-OFF contrast ratio \\
\hline AND & 4.9 \\
NAND & 7,4 \\
XNOR & 7.2 \\
NOR & 3.1 \\
\hline
\end{tabular}




\section{Conclusions}

A versatile all-optical logic gate based on the self-collimation effect in two dimensional photonic crystals is proposed. Numerical simulations are demonstrated successfully by using the finite difference time domain (FDTD) method which shows that the structure performs AND, NAND, $\mathrm{XNOR}$ and NOR logic functions. The designed structure can perform the logic gate functions in the same configuration only by adjusting the input power levels. It has simple geometric and clear operating principle. It operates at the optical communication wavelength of $1.5551 \mu \mathrm{m}$ and its ON-OFF contrast ratio is about $6 \mathrm{~dB}$. Because of the compactness, this device is suitable for optical computing and photonic integrated circuits.

Open Access This article is distributed under the terms of the Creative Commons Attribution License which permits any use, distribution, and reproduction in any medium, provided the original author(s) and source are credited.

\section{References}

[1] T. Yamamoto, E. Yoshida, and M. Nakazawa, "Ultrafast nonlinear optical loop mirror for demultiplexing $640 \mathrm{Gbit} / \mathrm{s}$ TDM signals," Electronics Letters, vol. 34, no. 10, pp. 1013-1014, 1998.

[2] T. Fjelde, A. Kloch, D. Wolfson, B. Dagens, A. Coquelin, I. Guillemot, F. Gaborit, F. Poingt, and M. Renaud, "Novel scheme for simple label-swapping employing XOR logic in integrated interferometric wavelength converter," IEEE Photonics Technology Letters, vol. 13, no. 7, pp. 750-752, 2001.

[3] J. P. Wang, B. S. Robinson, S. A. Hamilton, and E. P. Ippen, "Demonstration of $40 \mathrm{~Gb} / \mathrm{s}$ packet routing using all optical header processing," IEEE Photonics Technology Letters, vol. 18, no. 21, pp. 2275-2277, 2006.

[4] J. W. M. Menezes, W. B. Fraga, A. C. Ferreira, K. D. A. Saboia, A. F. G. F. Filho, G. F. Guimarães, J. R. R. Sousa, H. H. B. Rocha, and A. S. B. Sombra, "Logic gates based in two and three-modes nonlinear optical fiber couplers," Optical and Quantum Electronics, vol. 39, no. 14, pp. 1191-1206, 2007.
[5] A. Bogoni, L. Poti, R. Proietti, G. Meloni, F. Ponzini, and P. Ghelfi, "Regenerative and reconfigurable all-optical logic gates for ultra-fast applications," Electronics Letters, vol. 41, no. 7, pp. 435-436, 2005.

[6] K. H. Ahn, X. D. Cao, Y. Liang, B. C. Barnett, S. Chaikamnerd, and M. N. Islam, "Cascadability and functionality of all-optical low-birefringent non-linear optical loop mirror: experimental demonstration," Journal of Optical Society of America B, vol. 14, no. 5, pp. 1228-1236, 1997.

[7] K. S. Choi, Y. T. Byun, S. Lee, and Y. M. Jhon, "All-optical OR/NOR bi-functional logic gate by using cross-gain modulation in semiconductor optical amplifiers," Journal of the Korean Physical Society, vol. 56, no. 4, pp. 1093-1096, 2010.

[8] J. H. Kim, Y. M. Jhon, Y. T. Byun, S. Lee, D. H. Woo, and S. H. Kim, "All-optical XOR gate using semiconductor optical amplifiers without additional input beam," IEEE Photonics Technology Letters, vol. 14, no. 10, pp. 1436-1438, 2002.

[9] Z. H. Li and G. F. Li, "Ultrahigh speed reconfigurable logic gates based on four-wave mixing in a semiconductor optical amplifier," IEEE Photonics Technology Letters, vol. 18, no. 12, pp. 1341-1343, 2006.

[10] H. J. S. Dorren, X. L. Yang, A. K. Mishra, Z. G. Li, H. Ju, H. Waardt, G. D. Khoe, T. Simoyama, H. Ishikawa, H. Kawashima, and T. Hasama, "All-optical logic based on ultrafast gain and index dynamics in a semiconductor optical amplifier," IEEE Journal of Selected Topics in Quantum Electronics, vol. 10, no. 5, pp. 1079-1092, 2004.

[11] K. E. Stubkjaer, "Semiconductor optical amplifier-based all-optical gates for high-speed optical processing," IEEE Journal of Selected Topics in Quantum Electronics, vol. 6, no. 6, pp. 1428-1435, 2000.

[12] T. Yabu, M. Geshiro, T. Kitamura, K. Nishida, and S. Sawa, "All-optical logic gates containing a two-mode nonlinear waveguide," IEEE Journal of Quantum Electronics, vol. 38, no. 1, pp. 37-46, 2002.

[13] Y. D. Wu, "All-optical logic gates by using multibranch waveguide structure with localized optical nonlinearity," IEEE Journal of Selected Topics in Quantum Electronics, vol. 11, no. 2, pp. 307-312, 2005

[14] Y. D. Wu, T. T. Shih, and M. H. Chen, "New all-optical logic gates based on the local nonlinear Mach-Zehnder interferometer," Optics Express, vol. 16, no. 1, pp. 248-257, 2008.

[15]K. Y. Lee, Y. C. Yang, Y. J. Lin, W. Y. Lee, C. C. Lee, and S. H. Wong, "The designs of $4 \times 2$ encoder based on photonic crystals," in Proc. SPIE (Proceedings of 
OSA-IEEE Asia Communications and Photonics), vol. 7630, pp. 76300I-1-76300I-7, 2009.

[16] F. Cuesta-Soto, A. Martínez, J. García, F. Ramos, P. Sanchis, J. Blasco, and J. Martí, "All-optical switching structure based on a photonic crystal directional coupler," Optics Express, vol. 12, no. 1, pp. 161-167, 2004.

[17] M. Notomi, T. Tanabe, A. Shinya, E. Kuramochi, H. Taniyama, S. Mitsugi, and M. Morita, "Nonlinear and adiabatic control of high- $Q$ photonic crystal nanocavities," Optics Express, vol. 15, no. 26, pp. 17458-17481, 2007.

[18] P. Andalib and N. Granpayeh, "All-optical ultra compact photonic crystal AND gate based on nonlinear ring resonators," Journal of Optical Society of America B, vol. 26, no. 1, pp. 10-16, 2009.
[19] H. S. Kim, T. K. Lee, G. Y. Oh, D. G. Kim, and Y. W. Choi, "Analysis of all optical logic gate based on photonic crystals multimode interference," in Proc. SPIE, vol. 7606, pp. 76061F-1, 2010.

[20] X. S. Christina, A. P. Kabilan, and P. E. Caroline, "Ultra compact all optical logic gates using photonic band-gap materials," Journal of Nanoelectronics and Optoelectronics, vol. 5, no. 3, pp. 397-401, 2010.

[21] H. Kosaka, T. Kawashima, A. Tomita, M. Notomi, T. Tamamura, T. Sato, and S. Kawakami, "Self-collimating phenomena in photonic crystals," Applied Physics Letters, vol. 74, no. 9, pp. 1212-1214, 1999.

[22] D. Y. Zhao, J. Zhang, P. J Yao, and X. Y. Jiang, "Photonic crystal Mach-Zehnder interferometer based on self-collimation," Applied Physics Letters, vol. 90, no. 23, pp. 231114-1-231114-3, 2007. 